\title{
Magneto-transport mechanism of individual nanostructures via direct magnetoresistance measurement in situ SEM
}

\author{
Junwei Zhang, ${ }^{a}$ Yong Peng, ${ }^{a}$ Hongbin Ma, ${ }^{\mathrm{d}}$ Senfu Zhang, ${ }^{\mathrm{c}}$ Yang Hu, ${ }^{\mathrm{a}}$ Xue Zeng, ${ }^{\mathrm{e}}$ Xia \\ Deng, ${ }^{\mathrm{b}}$ Chaoshuai Guan, ${ }^{\mathrm{a}}$ Rongrong Chen, ${ }^{\mathrm{a}}$ Yue Hu, ${ }^{\mathrm{a}}$ Abdul Karim,,${ }^{\mathrm{a}, \mathrm{g}}$ Kun Tao, ${ }^{\mathrm{a}}$ \\ Mingjie Zhang, ${ }^{\mathrm{f}}$ Xixiang Zhang ${ }^{\mathrm{c}}$
}

${ }^{\mathrm{a}}$ Key Laboratory of Magnetism and Magnetic Materials of the Ministry of Education, School of Physical Science and Technology and Electron Microscopy Centre of Lanzhou University, Lanzhou University, Lanzhou 730000, P. R. China.

${ }^{\mathrm{b} S c h o o l}$ of Life Science and Electron Microscopy Centre of Lanzhou University, Lanzhou University, Lanzhou 730000, P. R. China.

'King Abdullah University of Science and Technology (KAUST), Physical Science and Engineering (PSE) Division, Thuwal 23955-6900, Saudi Arabia.

${ }^{\mathrm{d}}$ Qinghai Provincial Key Laboratory of New Light Alloys, Qinghai Provincial Engineering Research Center of High Performance Light Metal Alloys and Forming, Qinghai University, Xining 810016, PR China.

eSchool of Mathematics and Physics, Lanzhou Jiaotong University, Lanzhou, 730070, PR China.

${ }^{\mathrm{f}}$ Key Lab of Mineral Resources in Western China (Gansu), School of Earth Sciences, Lanzhou University, Lanzhou 730000, China.

gDepartment Physics, Karakorum International University Gilgit-Baltistan, 15100.

*Corresponding author: pengy@1zu.edu.cn 


\begin{abstract}
The accurate magnetoresistance measurement of individual nanostructures is essential and significant for not only enriching the fundamental knowledge of magneto-transport mechanism but also facilitating the designs of desired magnetic nanostructures for diverse technological applications. Herein, we report a deep investigation on the magneto-transport mechanism of single $\mathrm{CoCu} / \mathrm{Cu}$ multilayered nanowire via direct magnetoresistance measurement by using our invented magnetotransport instrument in-situ scanning electron microscopy (SEM). Off-axis electron holography experiments combined with micromagnetic simulation prove that the $\mathrm{CoCu}$ layers in $\mathrm{CoCu} / \mathrm{Cu}$ multilayered nanowires are formed a multi-domain structure, in which the alignment of magnetic moments is mainly determined by shape anisotropy. The MR of the single $\mathrm{CoCu} / \mathrm{Cu}$ multilayered nanowire is measured to be only $1.14 \%$ when the varied external field is applied along nanowire length axis, which matches with the theoretical prediction of Granular Films model. Density functional theory (DFT) calculations further disclose that spin-dependent scattering at the interface between magnetic and nonmagnetic layers is responsible for the intrinsic magneto-transport mechanism.
\end{abstract}

\title{
1. Introduction
}

The trend of miniaturization in micro-electronics makes nanowires (NWs) more and more prominent. Magnetic multilayered nanowires (MNWs) are one group of the nanowire family, which own giant magnetoresistance (GMR) effect and are mainly grown by using template method with electrodeposition technique ${ }^{1-3}$. Their magnetotransport behaviors are usually utilized for possible diverse nano-spintronic applications including magnetic memory, magnetic field sensor, logic devices, signal 
processing device, current driven microwave oscillators ${ }^{4-5}$. The accurate characterization of individual NWs rather than groups is thus essential and critical for the functionality and reliability of the NW-component magnetoelectronics and magnetosensors. However, the most magnetoresistance (MR) measurements are performed on the array of multilayered nanowires which are still embedded into template channels due to technology limitations ${ }^{6-7}$, by which the MR signal of a single nanowire is then estimated by averaging. It is therefore not accurate and includes uncertain contact resistance of electrodes. There are also a limited work on the MR measurement of real single nanowire by making the electrodes on a single MNW via e-beam lithography (EBL) technique ${ }^{8}$. This method unavoidably involves contact resistance or chemical contaminations during the complex EBL processes, which affects the result accuracy. ${ }^{9-10}$ This is the main reason why the reported work show huge scatter of the electrical and magneto-electrical properties of individual metallic nanowires.

Nanomanipulators in-situ scanning electron microscope (SEM) could be a good solution to sort out the accurate measurements of magneto-transport properties of a single nanowire. This technique has been employed to measure the electrical transport properties of various nanowires ${ }^{11-12}$, which can select contact points at will and easily realize the test of length-resistance relationship. In addition, the whole test process is dynamically visualized and the contact resistance can be effectively avoided by punching probes into nanowire body. However, beside our very early prototyped demonstration ${ }^{13}$, the measurement of magneto-transport property of a single magnetic nanowire by nanomanipulators in-situ SEM has not been reported yet as far as we have known. 
MR mechanism is considered to mainly generate from spin-dependent scattering of conducting electrons at interfaces between magnetic and non-magnetic layers. ${ }^{14}$ Magnetic state and external field direction also have an influence on the amplitude of MR effect. Therefore, the fully understanding of MR effect in individual magnetic multilayered NWs requires detailed information of interface, magnetic state and external field direction beside morphology, chemistry and geometry. Numerous experiments and model calculations have been already done to elucidate the impacts on the nanowire GMR. ${ }^{14-16}$ However, few studies focus on the interfacial structure at the atomic scale and magnetic state at nanoscale ${ }^{17-18}$ because of the requirement of extremely high spatial resolution. Aberration-corrected transmission electron microscopy (Cs-corrected TEM) and scanning transmission electron microscopy (Cscorrected STEM) equipped with biprism and Lorentz lens provide the detection possibility. ${ }^{19-21}$ The image function of both Cs-corrected TEM and Cs-corrected STEM has recently achieved a surprisingly high spatial resolution below $0.5 \AA$ and their chemical function (accessories of electron energy-loss spectroscopy (EELS) and energy-dispersive X-ray analysis (EDX)) has readily realized a direct imaging and electronic-state detection of individual atoms and even chemical bonds. ${ }^{22}$ The additional function of off-axis electron holography $(\mathrm{EH})^{23-25}$ and Lorentz mode reaches $\mathrm{a} \sim 1 \mathrm{~nm}$ high-resolution visualization of electromagnetic field in an isolated nanostructure.

In this work, we choose $\mathrm{CoCu} / \mathrm{Cu} \mathrm{MNW}$ as an example sample to contribute an exploration of MR mechanism of singe MNW via accurate characterization of its magneto-transport behavior in situ SEM by using our dedicated magneto-transport instrument (MTI) (see details in supplementary information S1). Off-axis EH method was employed to detect the magnetic state of the single $\mathrm{CoCu} / \mathrm{Cu} \mathrm{MNW}$. Theoretical 
three-dimensional (3D) distribution of magnetic moments was simulated by publicshared OOMMF software. Density functional theory (DFT) calculations on the base of experimental interface and magnetic state information were further carried out to disclose the intrinsic $\mathrm{MR}$ origin of the $\mathrm{CoCu} / \mathrm{Cu} \mathrm{MNW}$. To further verify the correctness of the measurement results, Granular Films model is employed. Our work should not only provide a mature technique to accurately measure magneto-transport properties of single NW in situ SEM, but also disclose the MR mechanism of magnetic MNW.

\section{Results and discussion}

\subsection{Structural and chemical information of the nanowires}

The magneto-electrical properties strongly depend upon the microstructure of the nanowires such as the diameter, the thickness of the non-magnetic and magnetic layers, and interfacial structure between layers and nanoparticles as well as chemical composition. Therefore, the structural and chemical information of the nanowires are firstly characterized. Figure 1a shows a representative SEM image of $\mathrm{CoCu} / \mathrm{Cu}$ MNWs, which were completely released from the channels of AAO template. Quantitative analysis discloses that the average diameter of the $\mathrm{CoCu} / \mathrm{Cu} \mathrm{MNWs}$ is approximately $52 \mathrm{~nm}$, and that of length is about $30 \mu \mathrm{m}$. TEM image (Fig. 1b) shows that individual MNWs clearly have a bamboo-like morphology, in which the black contrast sections are $\mathrm{CoCu}$ layers and those of grey are $\mathrm{Cu}$ layers (see more analysis in supplementary information S2 and chemical analysis below). This result confirms that the periodic multilayered structure of $\mathrm{CoCu} / \mathrm{Cu}$ nanowire has been successfully formed. Statistical analysis from numerous TEM images shows that the average thickness of the $\mathrm{CoCu}$ layers is about $124 \mathrm{~nm}$, and that of non-magnetic $\mathrm{Cu}$ layers for 
$\sim 14.2 \mathrm{~nm}$. It is then calculated that the length-to-diameter ratio $t / d$ of the $\mathrm{CoCu}$ layers ( $t$ and $d$ represent the thickness and diameter, respectively) is about 2.16, which is larger than $1,{ }^{26}$ demonstrating that the magnetization direction should prefer the length axis. In addition, the thickness of non-magnetic layer is a key factor to study the magneto-electrical properties of $\mathrm{MNWs}^{27}$, which is essential to be accurately measured. The thickness of the $\mathrm{Cu}$ layer (marked by yellow area) is thus detected under high-magnification TEM as shown in Fig. 1c, which gives an approximate 13.6 $\mathrm{nm}$. This thickness reveals that the interlayer exchange coupling between neighbored magnetic $\mathrm{CoCu}$ layers should become very weak and their magnetic moments between neighbored magnetic $\mathrm{CoCu}$ layers are also uncorrelated.
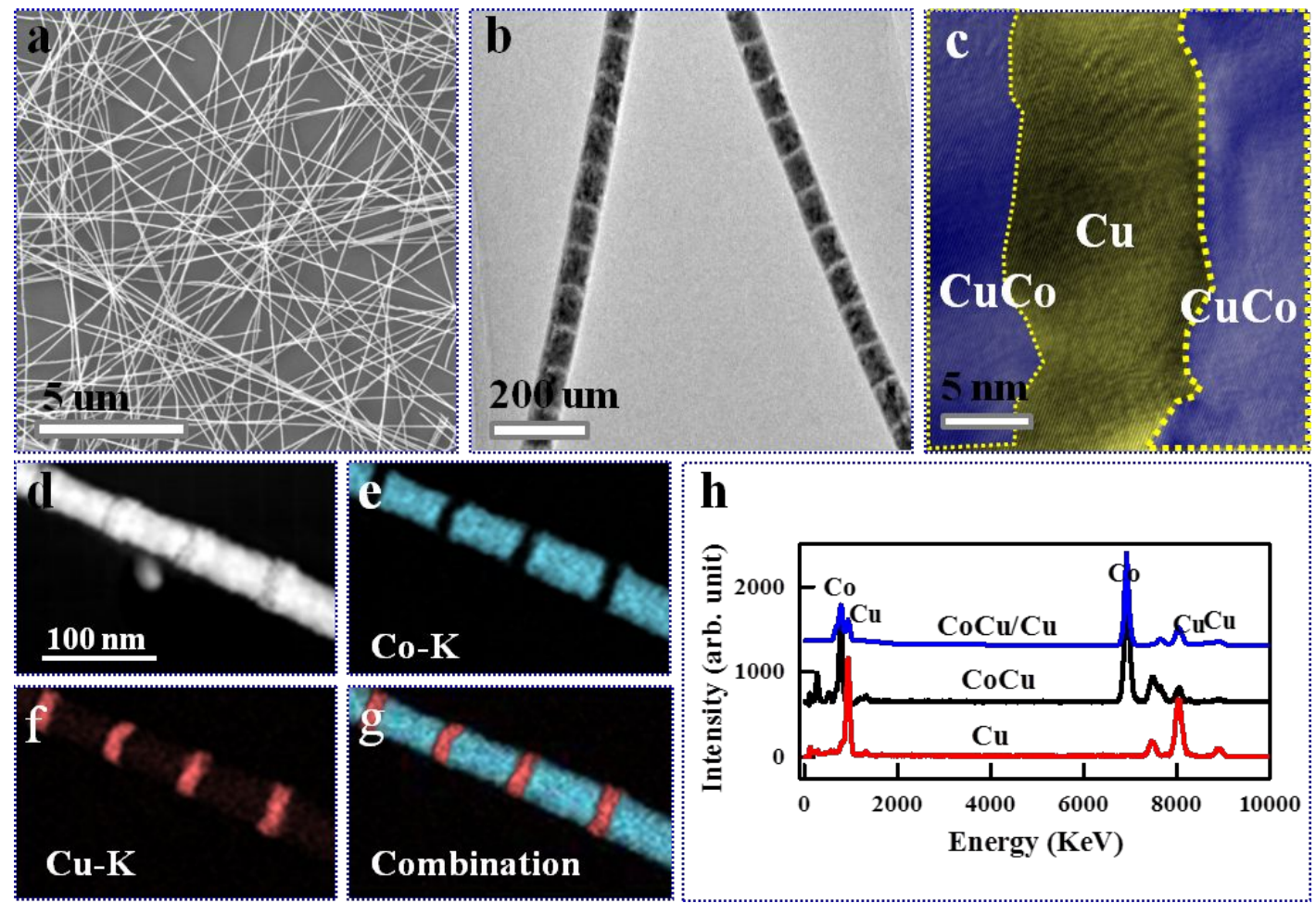

Figure 1. The morphology, crystal structure, chemical composition of the $\mathrm{CoCu} / \mathrm{Cu}$

MNWs analyzed by SEM and TEM. (a) SEM image of the nanowires dispersed on an $\mathrm{SiO}_{2}(200 \mathrm{~nm}) / \mathrm{Si}$ with free-standing formation after template dissolved; (b) TEM image of two nanowires with $55 \mathrm{~nm}$ uniform diameter; (c) A typical HRTEM of non-magnetic $\mathrm{Cu}$ layer; (d) HAADF-STEM image of MNW. (e) - (g) EDX elemental mappings of $\mathrm{Co} \mathrm{K} \alpha, \mathrm{Cu} \mathrm{K} \alpha$ and 
their combination, respectively. (h) EDX spectra measured from a bundle of $\mathrm{CoCu} / \mathrm{Cu}, \mathrm{CoCu}$ and $\mathrm{Cu}$ nanowires.

The chemical analysis was performed by using EDX and STEM mapping techniques. Fig. 1d shows the chosen single $\mathrm{CoCu} / \mathrm{Cu} \mathrm{MNW}$, of which elemental maps of $\mathrm{Co} \mathrm{Ka}$ and $\mathrm{Cu} \mathrm{Ka}$ are presented in Fig. 1e and 1f, respectively. Figure $1 \mathrm{~g}$ is their combination map, clearly showing a periodic structure of the blue $\mathrm{Co}$ and red $\mathrm{Cu}$ layers. Fig. 1e reveals that there is almost no $\mathrm{Co}$ element in the $\mathrm{Cu}$ layers, demonstrating a pure chemical composition. However, the signal of $\mathrm{Cu}$ element (Fig. 1f) exists in both $\mathrm{Co}$ and $\mathrm{Cu}$ layers. To quantify the proportion of the $\mathrm{Cu}$ impurity in the Co layers, additional single phase nanowires of $\mathrm{Cu}$ and $\mathrm{CoCu}$ were prepared (see supplementary information $\mathrm{S} 3$ for details). EDX spectra from the $\mathrm{CoCu} / \mathrm{Cu}, \mathrm{CoCu}$ and $\mathrm{Cu}$ nanowires were then measured to detect the chemical compositions as shown in Fig. 1h. As expected, $\mathrm{Cu}$ peaks appear in all spectra and Co peaks appear on both $\mathrm{CoCu} / \mathrm{Cu}$ and $\mathrm{CoCu}$ nanowires, revealing a pure $\mathrm{Cu}$ chemistry in the $\mathrm{Cu}$ layers of the multilayered nanowires. Quantitative analysis of the EDX spectrum from the single phase $\mathrm{CoCu}$ nanowires indicates a 92.1:7.9 atomic ratio of $\mathrm{Co} \mathrm{Cu}$, inferring a $\mathrm{Co}_{92.1} \mathrm{Cu}_{7.9} / \mathrm{Cu}$ composition of the multilayered nanowires. As our recent report ${ }^{1}$, the $\mathrm{CoCu}$ layers consist of $5 \mathrm{~nm} \mathrm{Cu}$ grains and $12 \mathrm{~nm} \mathrm{Co}$ grains, and both are fcc polycrystals with random orientations. Besides, the interface between $\mathrm{Co}$ and $\mathrm{Cu}$ grains is rough, which causes a large scatting with conduction electrons and reduces the magneto-resistance. Further investigation shows that the concentration of Co grains per unit volume is about $57.27 \%$ in the $\mathrm{CoCu}$ layers, of which detailed calculation is shown in supplementary information S4. 


\subsection{Resistance measurement of a single $\mathrm{CoCu} / \mathrm{Cu} \mathrm{MNW}$}

Electrical properties of the single $\mathrm{CoCu} / \mathrm{Cu} \mathrm{MNW}$ were measured by using dedicated MTI in situ SEM as illustrated in Fig. 2a. Figure $2 \mathrm{~b}$ shows a representative SEM image of the experimental set-up, where two conductive nichrome probes pierced the nanowire body to achieve a good ohmic contact. The measured resistance $\left(R_{\mathrm{e}}\right)$ includes the intrinsic resistance of $\mathrm{CoCu} / \mathrm{Cu}$ MNWs $\left(R_{\mathrm{i}}\right)$, the contact resistance $\left(R_{\mathrm{c}}\right)$ and the feed line resistance of circuitry $\left(R_{\mathrm{f}}\right)$. To remove the feed line resistance, the two tips were contacted to each other (Inset of Fig. 2c) and current vs applied voltage relationship was then recorded as shown in Fig. 2c. The inverse of the slope of this I-V curve gives a $60.3 \Omega$ feed line resistance.

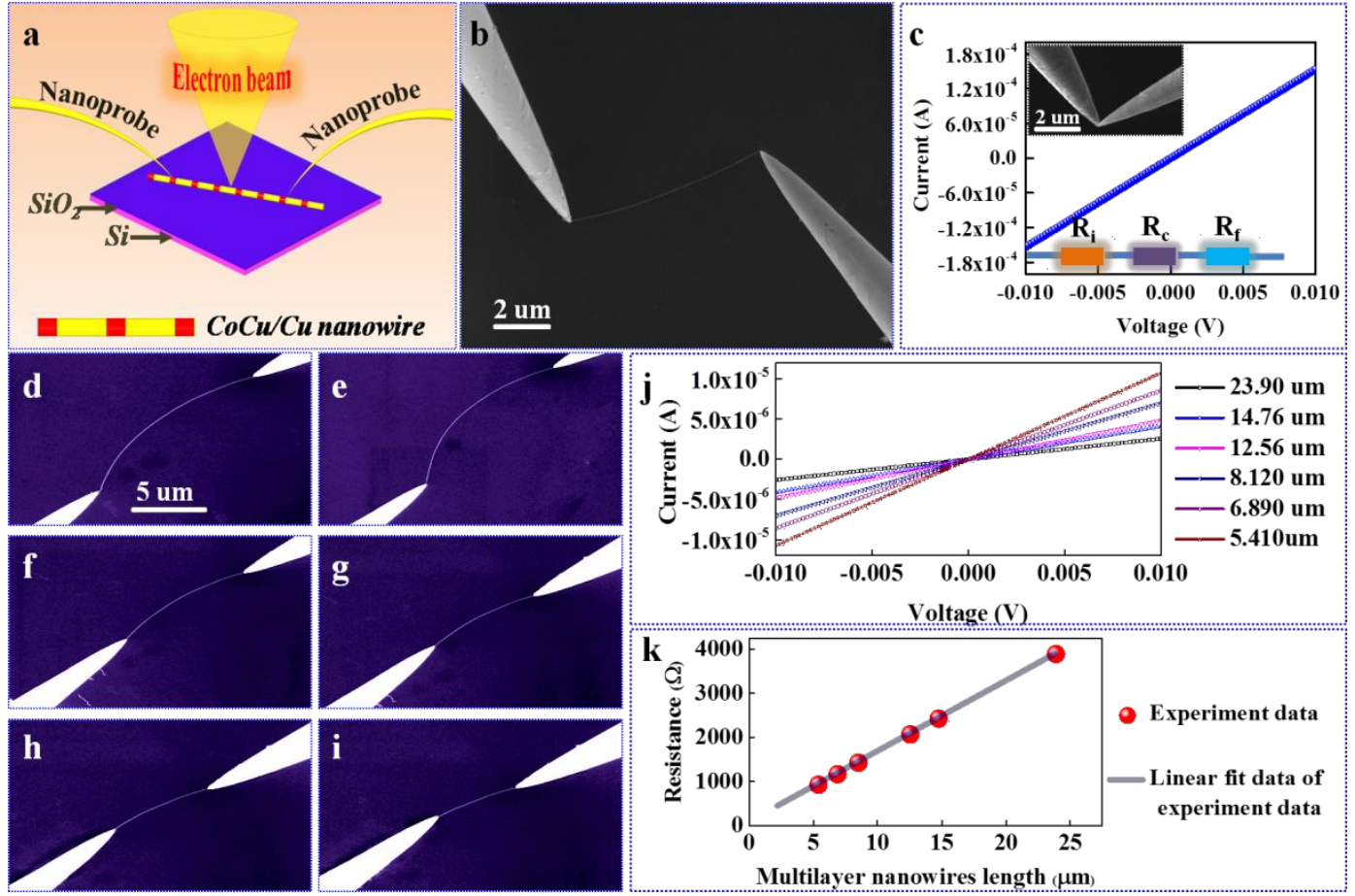

Figure 2 Resistance measurement of single periodic $\mathrm{CoCu} / \mathrm{Cu}$ MNW. (a) Schematic

illustration of the measurement setup, in which two conductive nichrome probes are tightly pressed into the nanowire body; (b) A representative SEM image of the experimental testing; (c) I-V behavior of the tip-tip circuit, as shown in the top-left illustration; (d) - (i) A series of SEM images of six measurement positions (length), which are for obtaining R-L curve; (j) 
The corresponding I-V behaviors at the 6 MNW lengths; (k) The plotted R-L curve, in which red dots are for the measured data and blue line is for the linear fit of the experimental data.

To further get the accurate values of $R_{\mathrm{i}}$ and $R_{\mathrm{c}}$, resistance-length $\left(R_{\mathrm{e}}-l\right)$ relationship were detected via the changes of touching positions of nanomanipulator tip. Fig. 2d-2i show the measured positions and Fig. $2 \mathrm{j}$ shows the corresponding $\mathrm{I}-\mathrm{V}$ curves of the six touching positions. Furthermore, $R_{\mathrm{e}}-l$ curve can be plotted according to these curves as shown in Fig. $2 \mathrm{k}$. It is found that $R_{\mathrm{e}}$ is a linear function of $l$ and thus the $R_{\mathrm{e}}-l$ curve is fitted with a linear function. The intercept $b$ of this curve, which is $62.8 \Omega$, is the sum of $R_{\mathrm{c}}$ and $R_{\mathrm{f}}$. Since $R_{\mathrm{f}}$ has been obtained above, the $R_{\mathrm{c}}$ is then calculated to be $R_{\mathrm{c}}=b-R_{\mathrm{f}}=2.5 \Omega$. This value demonstrates that the contact resistance is very small and the intrinsic $R_{\mathrm{i}}$ can be obtained by subtracting the intercept value of $62.8 \Omega$, which proves that our designed MTI in situ SEM is very accurate and reliable for detecting the electrical property of a single nanomaterial.

The resistivity $\rho$ of the MNW is further analyzed. It is noted that the $52 \mathrm{~nm}$ diameter of our MNW is larger than the free electron mean as reported in previous work. ${ }^{11-12,28}$ The apparent quantum confinement effect is negligible in the case of our $\mathrm{CoCu} / \mathrm{Cu} \mathrm{MNW}$ and the classical of resistance can be adapted as:

$$
R_{\mathrm{e}}=R_{i}+R_{c}+R_{f}=\frac{\rho}{A} l+b
$$

where A is the MWN's cross-sectional area. $\rho$ is then calculated to be $3.39 \times 10^{-7} \Omega \cdot \mathrm{m}$, which is higher than that of the standard $\mathrm{Co}$ and $\mathrm{Cu}$ bulk $\left(6 \times 10^{-8} \Omega \cdot \mathrm{m}^{29}\right.$ and $1.75 \times 10^{-8}$ $\Omega \cdot \mathrm{m}^{30}$ at the room temperature, respectively). Based on the structural and chemical information of MWN, this high resistivity is deduced to originate from the rough interface structure between $\mathrm{CoCu}$ layers and $\mathrm{Cu}$ layers (Fig. 1c), which may lead to an increase of electron scattering at the interface. Besides, the nanocrystalline size, the 
number of interface, and diameter of the nanowires also influence the resistivity.

\subsection{Magnetoresistance measurement of a single $\mathrm{CoCu} / \mathrm{Cu} \mathrm{MNW}$ in situ SEM}
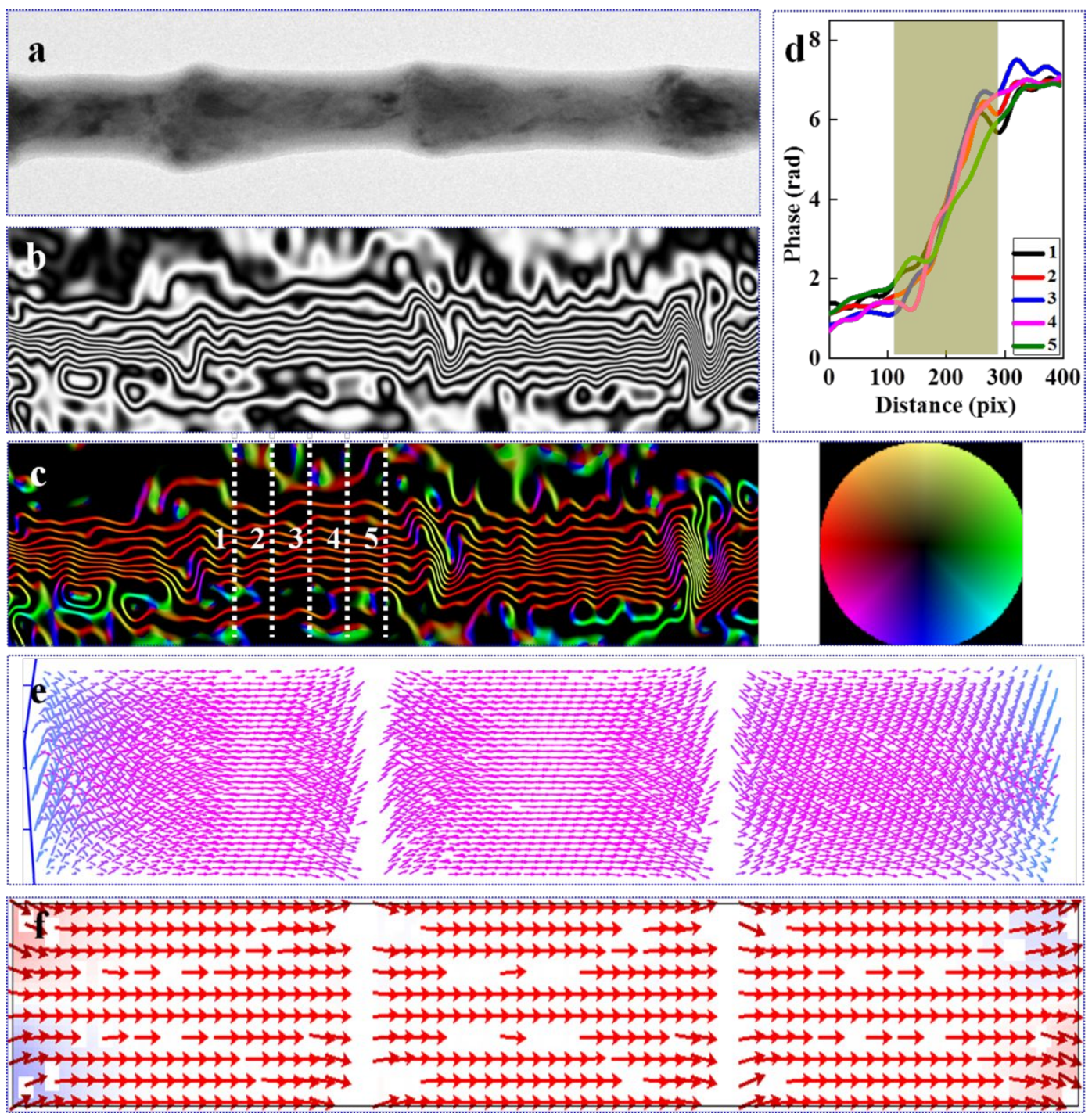

Figure 3. Distribution of magnetic moments in $\mathrm{CoCu} / \mathrm{Cu}$ MNW. (a) STEM image of the

chosen single MNW; (b) Magnetic flux image displayed by cosine of phase $\phi \mathrm{M}$ which has been amplified 10 times $(\cos (10 \phi \mathrm{M}))$; (c) Color-coded $\nabla \phi$ where the color hue and brightness represent $\operatorname{atan}(10 \nabla \phi)$ and $|\nabla \phi|$, respectively. (d) The 5 line profiles of magnetic phase marked by using white dotted line in (c); (e) Simulated 3D micromagnetic structures; (f) The corresponding 2D magnetic distribution. 
MR is known to relate to the distribution of the magnetic moments and external field direction. ${ }^{22}$ In order to correctly characterize the origin of magnetic moment distribution in the $\mathrm{CoCu} / \mathrm{Cu} \mathrm{MNW}$ at the remnant magnetic state, traditional VSM measurement and off-axis EH method were employed. Fig. S4 shows two hysteresis loops measured under the external field perpendicular and parallel to the MNW length axis, revealing that the easy axis of the MNW is along the MNW length axis. Fig. 3a shows a chosen single $\mathrm{CoCu} / \mathrm{Cu} \mathrm{MNW}$ used for off-axis $\mathrm{EH}$ detection at room temperature and Fig. $3 \mathrm{~b}$ presents its corresponding magnetic flux $\left(\operatorname{Cos}\left(10 \varphi_{M A G}\right)\right)$ image. It is clearly observed that the magnetic lines are mainly parallel to the MNW length axis and bend at the ends of each $\mathrm{CoCu}$ layer, revealing that the length axis of the single MNW is easy axis and the dipolar interaction between $\mathrm{CoCu}$ layers is weak. This observation is consistent with above structural and chemical analysis. According to above and our previous reported ${ }^{1}$ structural information, the $\mathrm{CoCu} / \mathrm{Cu} \mathrm{MNW}$ is polycrystalline and the magnetocrystalline anisotropy can be ignored. The magnetic flux image also demonstrates that the magnetic moment distribution of this $\mathrm{CoCu} / \mathrm{Cu}$ MNW is determined by its shape anisotropy rather than its magnetocrystalline anisotropy and dipolar interaction. To further determine the physical reason of magnetic moments distribution in $\mathrm{CoCu}$ layers, the $\varphi_{M A G}$ distribution in the forms of color-coded $\left(\nabla \varphi_{M A G}\right)$ and the profile of magnetic phase shift are carried out. ${ }^{31}$ Fig. 3c is the $\varphi_{M A G}$ distribution in the forms of color-coded $\left(\nabla \varphi_{M A G}\right)$ where the color hue and brightness represent the direction $\left(\operatorname{atan}\left(\nabla \varphi_{M A G}\right)\right)$ and intensity $\left(\left|\nabla \varphi_{M A G}\right|\right)$ of the inplane component of magnetic moment, respectively. It is observed that the magnetic moments mainly arrange along the nanowire length axis. However, the magnetic moments at some locations are disorderly arranged inside $\mathrm{CoCu}$ layers, revealing that the $\mathrm{CoCu}$ layers have a multi-domain structure. The profiles of phase shift as shown 
in Fig. 3d coming from the five lines in Fig. 3c (numbered as 1-5) further enlarge the turbulence of magnetic flux (especially rectangle region), double revealing the feature of multi-domain structure. It is known that a single crystal particle is typically in a single domain state when its radius is smaller than critical single domain radius. Theoretically, a single domain state can be maintained in a $12 \mathrm{~nm}$ single-crystalline Co particle under the remnant magnetic state, in which the direction of magnetic moments is determined by magnetocrystalline anisotropy. The random orientations of Co grains with tiny size in the magnetic $\mathrm{CoCu}$ layers in this work therefore induce the formation of multi-domain structure. This result proves that shape anisotropy is a key factor to determine the micromagnetic configuration of the $\mathrm{CoCu}$ layers in the $\mathrm{CoCu} / \mathrm{Cu} \mathrm{MNWs}$ rather than magnetocrystalline anisotropy.

Micromagnetic simulations by using OOMMF software were further used to verify this experimental observation, ${ }^{32}$ in which the sample model was constructed on the base of experimentally morphological geometries, crystal structures and chemical compositions (see more details in supplementary information S6). Figure 3e and $3 \mathrm{f}$ respectively show the simulated three-dimensional (3D) and 2D magnetic induction maps of the $\mathrm{CoCu} / \mathrm{Cu} \mathrm{MNW}$, clearly revealing that the magnetic moments of each magnetic layer are in a consistent state around MWN length axis and appear a curling state at both ends of each magnetic $\mathrm{CoCu}$ layer. This result further reveals that the shape anisotropy plays a dominant role in the alignment of magnetic moments when the length-to-diameter ratio $t / d$ of magnetic layer is larger than 1 .

The MR effect of individual $\mathrm{CoCu}$ MNWs was then measured by the dedicated MTI in-situ SEM (Fig. 4a). Fig. 4b shows a typical SEM image of practical setup, where two conductive nichrome tips stabbed into a $3.51 \mu \mathrm{m} \mathrm{CoCu} / \mathrm{Cu} \mathrm{MNW}$ and the MNW was immersed in a varied external field. Figure $4 \mathrm{c}$ shows the collected MR 
signal (based on standard MR formula of $M R=\left[\left(R_{H}-R_{\max }\right) / R_{\max }\right] \times 100 \%$ ) under 1500 Oe maximum field at room temperature. The MR value reaches the maximum value of $1.14 \%$ at about \pm 270 Oe and minimum value at saturation field, indicating a 270 Oe coercivity. This value is smaller than that of reported $\mathrm{CoCu} / \mathrm{Cu} \mathrm{MNWs}$ arrays $^{33}$, which is deduced that the single MNW escapes the neighbored magnetic interactions that exist in MNWs array from group measurements. Besides, above offaxis EH experimental results and micromagnetic simulations reveal that shape anisotropy plays a decisive role in the distribution of magnetic moments in the single $\mathrm{CoCu} / \mathrm{Cu} \mathrm{MNW}$, which also means that the MR of individual MNWs is dominated by anisotropic magnetoresistance (AMR). However, the detected MR effect of the single $\mathrm{CoCu} / \mathrm{Cu} \mathrm{MNW}$ is very small and the AMR should be almost zero when the direction of the applied magnetic field is parallel to the nanowire length axis ${ }^{3}$. Therefore, the MR of the single $\mathrm{CoCu} / \mathrm{Cu} \mathrm{MNW}$ should be governed by other mechanism. Density functional theory (DFT) calculations were employed to further understand the MR mechanism of the $\mathrm{CoCu} / \mathrm{Cu} \mathrm{MNW}$. Figure $4 \mathrm{~d}$ presents an atomic model of $\mathrm{Co}-\mathrm{Cu}$ interface along [111] direction according to our recent report of atomic packing analysis by $\mathrm{Cs}-\mathrm{STEM}^{1}$, in which $4 \times 4 \times 1 \mathrm{Cu}$ unit cell (marked by blue balls) and $4 \times 4 \times 1$ Co unit cell (marked by red balls) are consisted of. Figure $4 \mathrm{e}$ and $4 \mathrm{f}$ show the $3 \mathrm{D}$ and $2 \mathrm{D}$ charge redistributions, respectively, defined as $\rho_{\mathrm{Co} / \mathrm{Cu}}-\rho_{\mathrm{Cu}}-\rho_{\mathrm{Co}}$, where $\rho_{\mathrm{Co} / \mathrm{Cu}}$ is the charge density of the $\mathrm{Co} / \mathrm{Cu}$ system, $\rho_{\mathrm{Cu}}$ or $\rho_{\mathrm{Co}}$ is the charge density of the $\mathrm{Cu}$ or Co surface. The red color represents for the electron accumulation and the blue is for the electron depletion. The d orbital of Co located at the blue area and the p orbital of $\mathrm{Cu}$ at the red area in Fig. $4 \mathrm{f}$ indicate that the d orbital of Co loses electrons and the $\mathrm{p}$ orbital of $\mathrm{Cu}$ gains electrons at the interface. Due to the charge redistribution, $\mathrm{Cu}$ atoms at the interface have induced weak magnetic moments (see supplementary 
information S7 for the calculated data in detail). As illustrated in Fig. 4g, the $\mathrm{Cu}$ and Co atomic layers at the interface have different values of magnetic moments and the opposite direction under zero external magnetic field, which cause a large scattering of conduction electrons and exhibit a state of high resistance. When the applied magnetic field is big enough along the nanowire length axis, the magnetic moments at the interfaces are parallel to align along the length axis and the spin-dependent scattering of the conducting electrons is reduced, which appears a state of low resistance. These physical interactions are similar with those of in granular films system $^{14}$, therefore, the intrinsic MR mechanism of the single MNW comes from spin-dependent scattering at the interface between $\mathrm{CoCu}$ and $\mathrm{Cu}$ layer.

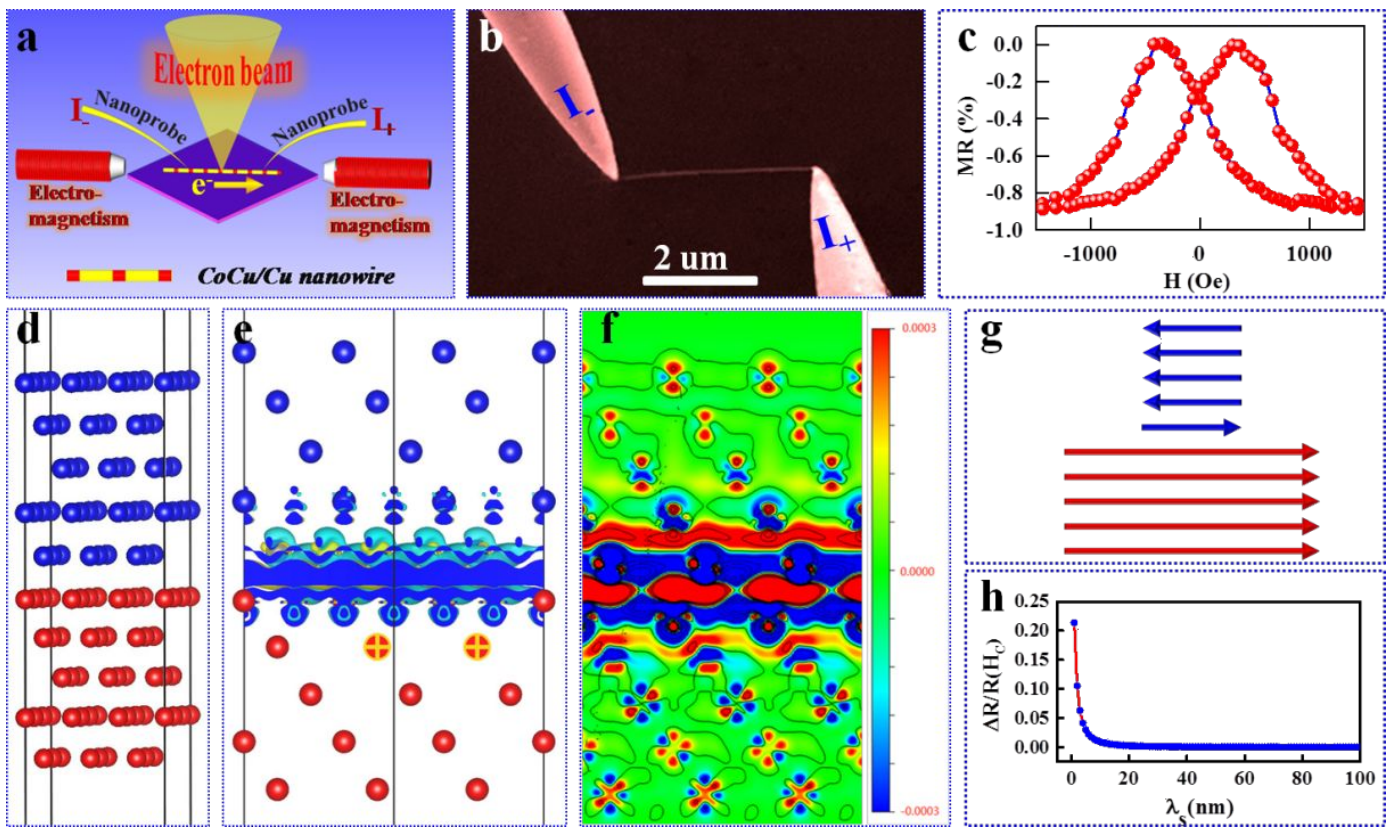

Figure 4. MR mechanism of single $\mathrm{CoCu} / \mathrm{Cu} \mathrm{MNW}$ by using MTI and DFT (a)

Schematic of measurement MR inside a SEM. (b) A representative SEM image of the experimental setup; (c) $\mathrm{MR}$ effect of individual $\mathrm{CoCu} / \mathrm{CuCo} \mathrm{MNWs}$ with the applied magnetic field parallel to the nanowire length axis; (d) Interfacial structure of $\mathrm{Co}$ and $\mathrm{Cu}$ along [011] orientation with a good lattice matching; (e) - (f) $3 \mathrm{D}$ and $2 \mathrm{D}$ charge redistributions, respectively, defined as $\rho_{\mathrm{Co} / \mathrm{Cu}}-\rho_{\mathrm{Cu}}-\rho_{\mathrm{Co}}$; (g) Illustration of magnetic moments at 
the interface; (h) MR as a function of the interface mean free path between $\mathrm{Co}$ and $\mathrm{Cu}$ grains.

In order to verify the correctness of our measurement, the Granular Films model was applied to analyze the intrinsic $\mathrm{MR}$ of the single $\mathrm{CoCu} / \mathrm{Cu} \mathrm{MNW}{ }^{14}$. In order to simplify the model, the tilts of magnetic moments at both ends of each magnetic $\mathrm{CoCu}$ layer are not taken into account. The MR is governed by eqn. 2 (see detailed derivations in supplementary information S8):

$$
\begin{aligned}
& \mathrm{MR}=-\frac{\rho_{2}^{2}}{\rho_{1}^{2}} \\
& \rho_{1}=\frac{C k}{n e^{2}}\left(\frac{1+P_{b}^{2}}{\lambda_{m}}+\frac{1-C}{C \lambda_{n m}}+\frac{3\left(1+P_{s}^{2}\right)}{r_{m} \lambda_{s}}\right) \\
& \rho_{2}=\frac{C k}{n e^{2}}\left(\frac{2 P_{b}}{\lambda_{m}}+\frac{6 P_{s}}{r_{m} \lambda_{s}}\right)
\end{aligned}
$$

where $C$ is the concentration of Co grains per unit volume, $57.27 \%$ for the $\mathrm{CoCu}$ layer; $d_{m}$ is the average diameter of $\mathrm{CoCu}$ layer; $P_{b}$ and $P_{\mathrm{s}}$ are the spin-dependent rations for scattering within the $\mathrm{CoCu}$ layers and at their surfaces, which are 0.2 and 0.52 , respectively; $\lambda_{m}=5 \mathrm{~nm}$ is the mean free path for Co grains and $\lambda_{\mathrm{nm}}=25 \mathrm{~nm}$ for the $\mathrm{Cu}$ matrix ${ }^{14}$. MR versus interface mean free paths $\left(\lambda_{\mathrm{s}}\right)$ is then plotted according to the eqn. 2 as shown in Fig. 4h. Thus, the MR of single $\mathrm{CoCu} / \mathrm{Cu}$ is achieved for a small $\lambda_{\mathrm{s}}$ (small interface roughness), indicating that spin dependent scattering mainly derives from the interfaces between $\mathrm{CoCu}$ and $\mathrm{Cu}$ layer. The $\mathrm{MR}$ is $1.11 \%$ when $\lambda_{\mathrm{nm}}$ $=9 \mathrm{~nm}$, which was close to the experimental value of $1.14 \%$. This result proves that the mechanism of magnetoresistance effect in individual $\mathrm{CoCu} / \mathrm{Cu} \mathrm{MNW}$ s is mainly derived from spin-dependent scattering of the interfaces between $\mathrm{CoCu}$ and $\mathrm{Cu}$ layers. Besides, it is demonstrated that our designing and manufacturing magneto-transport 
instrument can be used to precisely and simultaneously measure the magnetic transport properties of single nanomaterial in situ SEM.

\section{Conclusions}

In conclusion, we have investigated the $\mathrm{MR}$ origin of single magnetic $\mathrm{CoCu} / \mathrm{Cu}$ multilayered nanowires via precise measurement of magneto-transport property by our dedicated MTI in situ SEM. Structural and magnetic state analysis disclose that the polycrystalline $\mathrm{CoCu}$ layers composed of tiny grains form a multi-domain structure and the dipolar interaction between neighbored magnetic $\mathrm{CoCu}$ layers is very weak, whilst, the magnetic moments of each $\mathrm{CoCu}$ layer are mainly parallel to the nanowire length axis. The electron holography characterization further proves that shape anisotropy dominantly determines the magnetization configuration of magnetic layers in $\mathrm{CoCu} / \mathrm{Cu} \mathrm{MNW}$ when the length-to-diameter ratio of $\mathrm{CoCu}$ layers is larger than 1. Micromagnetic computational simulations double confirm the finding. The MR of individual $\mathrm{CoCu} / \mathrm{Cu} \mathrm{MNW}$ is experimentally measured to be $1.14 \%$, well matching with theoretical calculation of $1.11 \%$ by using Granular Films model. DFT calculations discover that the MR mechanism of the $\mathrm{CoCu} / \mathrm{Cu} \mathrm{MNW}$ originates from spin-dependent scattering at the interface between magnetic and non-magnetic layers. Our work should not only provide a visual evidence to understand the MR mechanism of magnetic MNW, but also demonstrate a novel method to precisely and simultaneously measure magneto-transport properties of single nanomaterial in situ SEM, which should be significant for the exploration of novel functional magnetic structures to satisfy the diverse applications in electronic nanodevices and nanosensors. 


\section{Methods}

Specimen Preparation. $\mathrm{CoCu} / \mathrm{Cu}$ MNWs were prepared by electrodeposition into anodic alumina (AAO) templates ${ }^{28,34-35}$ with $50 \mathrm{~nm}$ diameter pores. In a typical process, a gold layer was sputtered onto one side of the template to serve as the working electrode in a standard three-electrode electrochemical cell. A saturated calomel electrode was used as reference electrode and graphite electrode was used as counter electrode. The electrodeposition electrolyte used to deposit $\mathrm{CoCu}$ and $\mathrm{Cu}$ was composed of $0.5 \mathrm{~mol} \cdot \mathrm{L}^{-1} \mathrm{CoSO}_{4} \cdot 7 \mathrm{H}_{2} \mathrm{O}, 0.005 \mathrm{~mol} \cdot \mathrm{L}^{-1} \mathrm{CuSO}_{4} \cdot 7 \mathrm{H}_{2} \mathrm{O}$ and $0.6 \mathrm{~mol} \cdot \mathrm{L}^{-1}$ $\mathrm{H}_{3} \mathrm{BO}_{3}$. The electrodeposition potential was alternately pulsed between a constant $0.67 \mathrm{~V}$ for $30 \mathrm{~s}$ to deposit only $\mathrm{Cu}$ and $-1 \mathrm{~V}$ for $6 \mathrm{~s}$ to deposit $\mathrm{CoCu}$. The total deposition cycle numbers was 300 . The entire electrodeposition process was the same as our previous reported paper $^{1-2}$.

SEM and TEM Characterization. Magnetic $\mathrm{CoCu} / \mathrm{Co} \mathrm{MNWs}$ completely released from the AAO templates by using $0.1 \mathrm{M}$ sodium hydroxide solution were dispersed on an $\mathrm{SiO}_{2}(100 \mathrm{~nm}) / \mathrm{Si}$ and holey carbon-film-coated grids for SEM and STEM analysis, respectively. The morphology, atomic structure and chemistry of the MNWs were analyzed by a scanning electron microscope (SEM, Tescan MIRA 3, Czech) and an aberration-corrected scanning transmission electron microscopy (Cs-STEM, FEI Cubed Themis Z, USA) equipped with energy-dispersive X-ray analysis (Super-X), electron energy lose spectroscopy (EELS, Gatan GIF 965, USA), Lorentz lens and biprism, in which the electron biprism for off-axis EH can achieve $\sim 1 \mathrm{~nm}$ spatial resolution.

Magnetic Property Measurements and Electron Holography. The magnetic properties of the $\mathrm{CoCu} / \mathrm{Cu} \mathrm{MNWs}$ were carried out by using a vibrating sample magnetometer (VSM, Lakeshore, USA) and magnetic flux distribution was obtained 
by electron holography. Off-axis electron holography was carried out in Cs-STEM using an electron biprism at $180 \mathrm{~V}$, under which the amplitude and phase shift of electrons penetrated from sample were recorded. The phase shift is induced by the inplane component of magnetic and electrostatic potential, of which the magnetic caused phase shift can be obtained from the half of the signal difference between the paired holograms on the positive and negative side of the samples. All off-axis EH experiments were operated at zero magnetic field at room temperature.

Calculations methods. First principles calculations were performed in the framework of density functional theory (DFT) as implemented in the Vienna ab initio simulation package (VASP) ${ }^{36}$ with projector augmented wave (PAW) potentials and generalized gradient approximation (GGA) of Perdew-Burke-Ernzerhof (PBE). The energy cutoff for the plane-wave basis set was $520 \mathrm{eV}$, and total energy was converged to $10^{-5} \mathrm{eV}$. The crystal geometry was optimized without any symmetry constraints until all residual forces on each atom were less than $0.01 \mathrm{eV} \cdot \AA^{-1}$. The Brillouin zone sampling was done by using a Gamma point centered $5 \times 5 \times 1 k$-point mesh. A slab model using $4 \times 4 \times 1$ unit cell was constructed, which consists of $5 \mathrm{Cu}$ layers and $5 \mathrm{Co}$ layers in (011) direction (22 ̊̊ vacuum spacing).

Micromagnetic Simulations. The 3D magnetic moment distribution in single $\mathrm{CoCu} / \mathrm{Cu} \mathrm{MNW}$ was simulated by public OOMMF micromagnetic software. The geometrical parameters were defined on the basis of the measured results of STEM and TEM, and the magnetic parameters is obtained by the VSM measurement and the previous report.

MR Measurements. The MR measurements of the single $\mathrm{CoCu} / \mathrm{Cu} \mathrm{MNW}$ were done in situ SEM by using a dedicated MTI which was updated from our previous prototyped MTI. The electromagnet part in MTI was rotated to the nanowire long axis. 
After two conductive nichrome probes were tightly punched into the bottom ends of the single $\mathrm{CoCu} / \mathrm{Cu} \mathrm{MNW}$, a $0.2 \mathrm{~V}$ voltage was conducted to the $\mathrm{MNW}$ to measure its resistivity when a scanning magnetic field from 1500 Oe to -1500 Oe and opposite scan were applied to the single MNW.

\section{Acknowledgement}

This work was supported by National Natural Science Foundation of China (51771085, 51571104, 51801087 and 91962212), the Fundamental Research Funds for the Central Universities (lzujbky-2020-58).

\section{Author contributions}

J.W.Z. designed the experiments, prepared the specimens, characterised, analysed and interpreted all data, and wrote the paper. Y.P. designed the experiments, provided overall guidance, supervised the TEM measurements, characterised, analysed and interpreted the data, and wrote the paper. H. M. grew the $\mathrm{CoCu} / \mathrm{Cu}$ magnetic nanowires and measured the MR of single nanowire. X. Z. and X. D. performed the TEM measurements together. R. C. performed the DFT calculation. J. Z and Y. P. drafted the paper and all authors commented on the manuscript. S. F. Z., C. S. G., Y. H. and A. K. discussed the data. K.T. performed the DFT calculation and wrote the DFT part of the manuscript. X.X.Z. analysed the magnetic data and discussed the content. J. Z. and H. M. contributed equally to this research. 


\section{Reference}

1. Zhang, J.; Chen, R.; Li, X.; Peng, Y.; Ma, H.; Hu, Y.; Zeng, X.; Deng, X.; Guan, C.; Hu, Y.; Zhang, M.; Karim, A.; Tao, K.; Zhang, X., Interfacial Roughness Facilitated by Dislocation and a Metal-Fuse Resistor Fabricated Using a Nanomanipulator. ACS Applied Materials \& Interfaces 2020.

2. Zhang, J.; Ma, H.; Zhang, S.; Zhang, H.; Deng, X.; Lan, Q.; Xue, D.; Bai, F.; Mellors, N. J.; Peng, Y., Nanoscale characterisation and magnetic properties of Co81Cu19/Cu multilayer nanowires. Journal of Materials Chemistry C 2015, 3 (1), 85-93.

3. Ohgai, T.; Hoffer, X.; Fábián, A.; Gravier, L.; Ansermet, J.-P., Electrochemical synthesis and magnetoresistance properties of $\mathrm{Ni}, \mathrm{Co}$ and $\mathrm{Co} / \mathrm{Cu}$ nanowires in a nanoporous anodic oxide layer on metallic aluminium. Journal of Materials Chemistry 2003, 13 (10), 2530-2534.

4. Kozlov, S. N.; Skryabina, O. V.; Egorov, S. V.; Golovchanskiy, I. A.; Klimenko, A. A.; Napolskii, K. S.; Stolyarov, V. S., Magnetoresistance of a single polycrystalline nickel nanowire. Journal of Applied Physics 2019, 125 (6), 063902.

5. Krimpalis, S.; Dragos, O. G.; Grigoras, M.; Lupu, N.; Chiriac, H., Magnetoresistance and spin transfer torque in electrodeposited $\mathrm{NiFe} / \mathrm{Cu}$ multilayered nanowires. Journal of Advanced Research in Physics 2010, 1 (2).

6. Tang, X.-T.; Wang, G.-C.; Shima, M., Layer thickness dependence of CPP giant magnetoresistance in individual $\mathrm{Co} \mathrm{Ni} \mathrm{Cu}$ multilayer nanowires grown by electrodeposition. Physical Review B 2007, 75 (13), 134404.

7. Krimpalis, S.; Dragos, O.-G.; Moga, A.-E.; Lupu, N.; Chiriac, H., Magnetization processes in electrodeposited $\mathrm{NiFe} / \mathrm{Cu}$ multilayered nanowires. Journal of Materials Research 2011, 26 (9), 1081-1090. 
8. Li, P.; Wu, W.; Wen, Y.; Zhang, C.; Zhang, J.; Zhang, S.; Yu, Z.; Yang, S. A.; Manchon, A.; Zhang, X. X., Spin-momentum locking and spin-orbit torques in magnetic nano-heterojunctions composed of Weyl semimetal WTe2. Nat Commun 2018, 9 (1), 3990.

9. Dubois, S.; Beuken, J.; Piraux, L.; Duvail, J.; Fert, A.; George, J.; Maurice, J., Perpendicular giant magnetoresistance of $\mathrm{NiFe} / \mathrm{Cu}$ and $\mathrm{Co} / \mathrm{Cu}$ multilayered nanowires. Journal of magnetism and magnetic materials 1997, 165 (1-3), 30-33.

10. Huang, X.; Tan, L.; Cho, H.; Stadler, B. J., Magnetoresistance and spin transfer torque in electrodeposited $\mathrm{Co} / \mathrm{Cu}$ multilayered nanowire arrays with small diameters. Journal of Applied Physics 2009, 105 (7), $07 \mathrm{D} 128$.

11. Peng, Y.; Cullis, T.; Inkson, B., Accurate electrical testing of individual gold nanowires by in situ scanning electron microscope nanomanipulators. Applied Physics Letters 2008, 93 (18), 183112.

12. Peng, Y.; Cullis, T.; Luxmoore, I.; Inkson, B., Electrical properties of individual $\mathrm{CoPt} / \mathrm{Pt}$ multilayer nanowires characterized by in situ SEM nanomanipulators. Nanotechnology 2011, 22 (24), 245709.

13. Elawayeb, M.; Peng, Y.; Briston, K. J.; Inkson, B. J., Electrical properties of individual $\mathrm{NiFe} / \mathrm{Pt}$ multilayer nanowires measured in situ in a scanning electron microscope. Journal of Applied Physics 2012, 111 (3), 034306.

14. Zhang, S., Theory of giant magnetoresistance in magnetic granular films. Applied physics letters 1992, 61 (15), 1855-1857.

15. Prieto, A. G.; Fdez-Gubieda, M., Influence of the interface on the magnetic anisotropy of $\mathrm{CoCu}$ granular alloys. Physica B: Condensed Matter 2004, 354 (1-4), 92-97. 
16. Prieto, A. G.; Fdez-Gubieda, M. L.; Chaboy, J.; Laguna-Marco, M. A.; Muro, T.; Nakamura, T., -Interface effects on the magnetic moment of $\mathrm{Co}$ and $\mathrm{Cu}$ in $\mathrm{CoCu}$ granular alloys. Physical Review B 2005, 72 (21).

17. Gan, W.; Chandra Sekhar, M.; Wong, D.; Purnama, I.; Chiam, S.; Wong, L.; Lew, W., Multi-vortex states in magnetic nanoparticles. Applied Physics Letters 2014, 105 (15), 152405.

18. Bryan, M.; Bance, S.; Dean, J.; Schrefl, T.; Allwood, D., Transverse and vortex domain wall structure in magnetic nanowires with uniaxial in-plane anisotropy. Journal of Physics: Condensed Matter 2011, 24 (2), 024205.

19. Beeli, C.; Doudin, B.; Ansermet, J.-P.; Stadelmann, P., Study of Co, Ni and $\mathrm{Co} / \mathrm{Cu}$ nanowires: magnetic flux imaging by off-axis electron holography. Journal of magnetism and magnetic materials 1996, 164 (1-2), 77-90.

20. Akhtari-Zavareh, A.; Carignan, L.; Yelon, A.; Ménard, D.; Kasama, T.; Herring, R.; Dunin-Borkowski, R. E.; McCartney, M.; Kavanagh, K., Off-axis electron holography of ferromagnetic multilayer nanowires. Journal of applied physics 2014, $116(2), 023902$.

21. Cantu-Valle, J.; Barriga-Castro, E. D.; Vega, V.; García, J.; Mendoza-Reséndez, R.; Luna, C.; Prida, V. M.; Nielsch, K.; Mendoza-Santoyo, F.; Jose-Yacaman, M., Quantitative magnetometry analysis and structural characterization of multisegmented cobalt-nickel nanowires. Journal of Magnetism and Magnetic Materials 2015, 379, 294-299.

22. Reyes, D.; Biziere, N.; Warot-Fonrose, B.; Wade, T.; Gatel, C., Magnetic configurations in $\mathrm{Co} / \mathrm{Cu}$ multilayered nanowires: evidence of structural and magnetic interplay. Nano letters 2016, 16 (2), 1230-1236. 
23. Rodríguez, L. A.; Bran, C.; Reyes, D.; Berganza, E.; Vázquez, M.; Gatel, C.; Snoeck, E.; Asenjo, A., Quantitative nanoscale magnetic study of isolated diametermodulated FeCoCu nanowires. ACS nano 2016, 10 (10), 9669-9678.

24. Midgley, P. A.; Dunin-Borkowski, R. E., Electron tomography and holography in materials science. Nature materials 2009, 8 (4), 271.

25. Gan, Z.; Gu, M.; Tang, J.; Wang, C.-Y.; He, Y.; Wang, K. L.; Wang, C.; Smith, D. J.; McCartney, M. R., Direct mapping of charge distribution during lithiation of Ge nanowires using off-axis electron holography. Nano letters 2016, 16 (6), 3748-3753.

26. Tang, X. Magnetotransport and magnetization reversal of electrodeposited multilayer nanowires. Rensselaer Polytechnic Institute, 2007.

27. Kashi, M. A.; Ghaffari, M.; Torshizi, F., Structural and magnetic tunability of $\mathrm{Co} / \mathrm{Cu}$ multilayer nanowires induced by electrolyte acidity and spacer layer thickness. Journal of Alloys and Compounds 2019, 153087.

28. Peng, Y.; Cullis, T.; Möbus, G.; Xu, X.; Inkson, B., Nanoscale characterization of CoPt/Pt multilayer nanowires. Nanotechnology 2007, 18 (48), 485704.

29. Datta, M.; Osaka, T.; Schultze, J. W., Microelectronic packaging. CRC press: 2004.

30. Hanaoka, Y.; Hinode, K.; Takeda, K. i.; Kodama, D., Increase in electrical resistivity of copper and aluminum fine lines. Materials transactions 2002, 43 (7), 1621-1623.

31. Murakami, Y.; Tanigaki, T.; Sasaki, T.; Takeno, Y.; Park, H.; Matsuda, T.; Ohkubo, T.; Hono, K.; Shindo, D., Magnetism of ultrathin intergranular boundary regions in Nd-Fe-B permanent magnets. Acta Materialia 2014, 71, 370-379.

32. Carlton, D. B.; Emley, N. C.; Tuchfeld, E.; Bokor, J., Simulation studies of nanomagnet-based logic architecture. Nano letters 2008, 8 (12), 4173-4178. 
33. Wang, J.-Q.; Xiao, G., Transition-metal granular solids: Microstructure, magnetic properties, and giant magnetoresistance. Physical Review B 1994, 49 (6), 3982.

34. Schlörb, H.; Haehnel, V.; Khatri, M. S.; Srivastav, A.; Kumar, A.; Schultz, L.; Fähler, S., Magnetic nanowires by electrodeposition within templates. Phys. Status Solidi B 2010, 247 (10), 2364-2379.

35. Wade, T. L.; Wegrowe, J.-E., Template synthesis of nanomaterials. The European Physical Journal-Applied Physics 2005, 29 (1), 3-22.

36. Kresse, G.; Furthmüller, J.; Hafner, J., Theory of the crystal structures of selenium and tellurium: the effect of generalized-gradient corrections to the local-density approximation. Physical Review B 1994, 50 (18), 13181. 